\section{AB1259 EVALUATION OF ENALAPRIL ON ARTERIAL STIFFNESS IN RHEUMATOID ARTHRITIS IN A RANDOMISED CLINICAL TRIAL}

Felipe Perez-Vazquez ${ }^{1}$, Sergio Duran Barragann ${ }^{2}$, Eduardo Gomez-Bañuelos ${ }^{1,3}$ Efrain Chavarria Avila ${ }^{1}$, Carlos Ramos Becerra ${ }^{4}$, Gustavo-Ignacio Diaz-Rubio ${ }^{5}$, Mario Salazar-Paramo ${ }^{4}$, Fernando Grover Páez ${ }^{4}$, Arnulfo Hernán Nava-Zavala ${ }^{4}$, Ernesto G. Cardona-Muñoz ${ }^{4}$, David Cardona Müller ${ }^{4}$, Rosa E. NavarroHernández ${ }^{5}$, Valeria-Nicole Mera-Riofrio ${ }^{5}$, Natalia Prado-Bachega ${ }^{5}$, Monica Vazquez-Del-Mercado ${ }^{5} .{ }^{1}$ Instituto de Investigación en Reumatología y del Sistema Músculo Esquelético, Departamento de Biología Molecular y Genómica, Guadalajara, Mexico; ${ }^{2}$ Hospital Civil Dr. Juan I. Menchaca, Servicio de Reumatología PNPC 004086 CONACyT, Guadalajara, Mexico; ${ }^{3}$ Universidad de Guadalajara, Centro Universitario de Ciencias de la Salud, Instituto de Investigación en Reumatología y del Sistema Músculo Esquelético, Departamento de Biología Molecular y Genómica, Guadalajara, Mexico; ${ }^{4}$ Universidad de Guadalajara, Centro Universitario de Ciencias de la Salud. Instituto de Terapéutica Experimental y Clínica, Departamento de Fisiología, Guadalajara, Jalisco, México, Guadalajara, Mexico; ${ }^{5}$ Universidad de Guadalajara, Centro Universitario de Ciencias de la Salud, Instituto de Investigación en Reumatología y del Sistema Músculo Esquelético, Departamento de Biología Molecular y Genómica, Guadalajara, Mexico

Background: This is the first study where suboptimal doses of enalapril proved to be successful for the reduction of arterial stiffness in in a clinical trial of rheumatoid arthritis (RA) patients.

Objectives: The aim of this study was to analyze the effect of enalapril on arterials stiffness through the evaluation of subclinical parameters in RA patients without traditional cardiovascular risk factors or previous comorbidities. Methods: Fifty three patients were enrolled in a clinical, randomized, closed-label trial. The subjects were randomly assigned into two groups: One receiving $5 \mathrm{mg}$ of enalapril (27) and $5 \mathrm{mg}$ of placebo (26), both twice a day. Clinical assessment included a structured questionnaire to gather demographic and clinical variables, including disease duration, treatment, disease activity, laboratory, anthropometric measurements and determination of cardiovascular parameters [carotid femoral pulse wave velocity (cfPWV) (1), cardio-ankle vascular index (CAVI) (2), carotid intima media thickness (cIMT), carotid artery distensibility (3), Young's incremental elastic modulus (Einc)]. The whole set of evaluations were analyzed at the baseline and at the end of 12 weeks of intervention.

Results: A significant reduction in delta CAVI of 0.21 in the enalapril intervention group was found. In contrast, an increase of 0.39 was observed in the placebo group. The delta CAVI reduction was not influenced by age or peripheral systolic blood pressure (pSBP).

Conclusion: Enalapril seems to be effective in CAVI reduction in RA patients. The effect of enalapril intervention on arterial stiffness translated to the clinical context, might be interpreted as a reduction of 6.4 years of arterial aging.

\section{REFERENCES}

[1] Vazquez-Del Mercado, M., et al., Disease duration of rheumatoid arthritis is a predictor of vascular stiffness: a cross-sectional study in patients without known cardiovascular comorbidities: A STROBE-compliant article. Medicine (Baltimore), 2017. 96(33): p. e7862.

[2] Saiki, A., et al., The Role of a Novel Arterial Stiffness Parameter, CardioAnkle Vascular Index (CAVI), as a Surrogate Marker for Cardiovascular Diseases. J Atheroscler Thromb, 2016. 23(2): p. 155-68.

[3] Vazquez-Del Mercado, M., et al., Subclinical parameters of arterial stiffness and arteriosclerosis correlate with QRISK3 in systemic lupus erythematosus. PLoS One, 2018. 13(12): p. e0207520.

Disclosure of Interests: None declared

DOI: 10.1136/annrheumdis-2019-eular.8241

\section{AB1260 CHARACTERISTICS OF METABOLIC SYNDROME IN AN ASIAN COHORT OF PATIENTS WITH RHEUMATOID ARTHRITIS}

Claire Teo Min-LI' Elampirai Elangovann², Chia Mun Woo ${ }^{3}$, Chien Joo Lim³ ${ }^{3}$, Khai Pang Leong ${ }^{1}$, Ee Tzun Koh ${ }^{1} .{ }^{1}$ Tan Tock Seng Hospital, Rheumatology, Allergy and Immunology, Singapore, Singapore; ${ }^{2} \mathrm{MOH}$ Holdings Pte Ltd, Singapore, Singapore; ${ }^{3}$ Tan Tock Seng Hospital, CMB - Clinical Research and Innovation Office, Singapore, Singapore

Background: Metabolic syndrome (MS), the concurrence of hypertension, abdominal obesity, dyslipidemia and glucose intolerance, is an important risk factor for type II diabetes mellitus (T2DM) and cardiovascular disease
(CVD). CVD is a leading cause of morbidity and mortality in patients with rheumatoid arthritis (RA). Studies in RA suggest that inflammation and glucocorticoid use predispose to MS, and that MS is associated with active disease.

Objectives: To investigate the prevalence and characteristics of MS in our cohort of RA patients.

Methods: A total of 409 patients who fulfilled the 1987 ACR criteria for RA and with complete data for classification of MS were included. MS was defined according to the Adult Treatment Panel III report of the National Cholesterol Education Programme (NCEP/ATP III). Information related to demographics, disease onset, serologies, disease activity and treatment was collected. Independent t-test or Mann-Whitney $U$ test was used to compare continuous quantitative data, while Pearson Chi-square or Fisher Exact test for categorical data. Logistic regression was used to identify factors associated with of MS. All the statistical tests were two sided, and $p$ value $<0.05$ was considered as statistically significant.

Results: Fifty-two patients (12.7\%) fulfilled the criteria for MS. Most of the patients in the cohort were female $(85.3 \%)$ and non-smokers $(84.6 \%)$. The mean age was $60.5 \pm 11.09$ years and mean BMI $23.67 \pm 5.06$. The patients with MS were significantly older (age $64.21+8.63$ vs $59.96 \pm 11.32$, $\mathrm{p}=0.010$ ). The mean disease duration was $14.09 \pm 9.74$ years. Rheumatoid factor and anti-cyclic citrullinated peptide antibody were present in $80.0 \%$ and $95.1 \%$ respectively. $4.4 \%$ of them had a history of CVD or stroke/ transient ischaemic attack but the prevalence was the same in patients with and without MS. Comparing the two groups, there was no significant difference in the history of ever-smoking ( $15.4 \%$ vs $15.4 \%, p=0.699)$, disease duration ( $16.44 \pm 10.11$ vs $13.72 \pm 9.66$ years, $p=0.060)$, cumulative glucocorticoid use $(p=0.649)$, DAS28 score $(2.12 \pm 1.09$ vs $2.11 \pm 1.24$ $\mathrm{p}=0.458)$ and $\mathrm{HAQ}$ score $(0.13$ vs $0.13, \mathrm{p}=0.767)$. There was also no significant difference in the use of methotrexate, leflunomide or biologics. The commonest MS criteria fulfilled were hypertension (88.5\%) and dyslipidaemia (84.6\%). $46.2 \%$ of the patients with MS had T2DM. Males were less likely to have MS than females [adj. OR $(95 \% \mathrm{Cl}): 0.039(0.005$, 0.292), $\mathrm{p}=0.002$.

Conclusion: MS is found in $12.7 \%$ of our RA patients and they were significantly older than those without MS. In our study, MS does not have a significant association with RA disease activity, disease duration, glucocorticoid usage or disease modifying anti-rheumatic treatment.

\section{REFERENCES}

[1] Rostom S, et al. Metabolic syndrome in rheumatoid arthritis: case control study. BMC Musculoskeletal Disorders. 2013 Apr 26;14:147.

[2] Tantayakom P, et al. Metabolic syndrome is associated with disease activity in patients with rheumatoid arthritis. Joint Bone Spine. 2016 Oct;83 (5):563

Disclosure of Interests: None declared DOI: 10.1136/annrheumdis-2019-eular.3070

\section{AB1261 CADMIUM TOXICITY AS A PROBABLE CAUSE OF OSTEOPENIA IN ADOLESCENTS AND ITS RELATION TO BAD DIETARY HABITS}

Adel Elbeialy ${ }^{1}$, Hesham Eldesouky ${ }^{2} .{ }^{1}$ Al-Azhar Faculty of Medicine, Rheumatology, Cairo, Egypt, ${ }^{1} \mathrm{Al}$-Azhar Faculty of Medicine, Rheumatology, Cairo, Egypt

Background: Cadmium is a naturally occurring minor element; it has been recognized as an occupational health hazard for many decades. Water and food are the main source of environmental cadmium exposure in non-smokers in most parts of the world. Cadmium accumulates gradually in the human body, where it gives rise to a number of adverse health effects and especially to kidney and bone. Several studies have addressed a possible association between long-term low-level environmental cadmium exposure and osteoporosis. Osteoporosis is a large and escalating public health problem.

Objectives: This study was conducted to assess the bone mineral status in secondary school students in Egypt and to measure cadmium level in their blood and urine and possible relationship between cadmium retention and bone mineral abnormalities as well as its consumption from some food and drinks commonly utilized by those students.

Methods: Two hundred secondary school students from different secondary schools in Egypt were included in this study $(100$ males, and 100 females). Bone mineral status was assessed in the 200 students by Quantitative Ultrasonography (QUS) of the calcaneus using the ultrasound bone densitometer unit PEGASUS PRESTIGE, OSTEOMED, FRANCE. Students with abnormal bone mineral status ( $T$ score $<-1$ ) were considered osteopenic, data obtained from this osteopenic group were compared 
to those from a group of apparently healthy students with normal $T$ score ( $T$ score $\geq-1$ ).

Results: Osteopenic group were 52 students (26\%); 16 males and 36 females, their mean age was $15.46 \pm 0.40$ years. Cadmium level in blood and urine was significantly higher in osteopenic group. Interpretation of dietary habits in the osteopenic and control groups revealed that carbonated beverages, potatoes chips, corn snacks intake were significantly increased in osteopenic group, whereas no significant difference was detected in milk, tea, and coffee intake. T score was negatively correlated with blood cadmium, urine cadmium, as well as carbonated beverages, potatoes chips, corn snacks. Cadmium concentrations in tap water as well as in commercial mineral water were negligible, but its concentration in carbonated beverages, potatoes chips, corn snacks was relatively high

Conclusion: Osteopenia and osteoporosis are not uncommon problem among secondary school students in Egypt. Cadmium exposure, evident by high blood and urinary levels, is a risk factor for development of low BMD. Fault dietary habits, including increased carbonated beverages, potatoes chips, and corn snacks intake, contributes to the occurrence of osteopenia.

\section{REFERENCES}

[1] Abdel-Haleem AS, Sroor A, El-Bahi SM, et al.: Heavy metals and rare earth elements in phosphate fertilizer components using instrumental neutron activation analysis. Appl Radiat Isot 55:569-573; 2001.

[2] Abdel-Sabour MF: Cadmium status in Egypt. J Environ Sci (China) 13:351-360; 2001

[3] Abou-Arab AA and Abou Donia MA: Heavy metals in Egyptian spices and medicinal plants and the effect of processing on their levels. J Agric Food Chem 48:2300-2304; 2000.

[4] Åkesson A, Bjellerup P, LundhT, et al.,: Cadmium-Induced Effects on Bone in a Population-Based Study of Women. Environmental Health Perspectives 116 (6): 830-834; 2006.

Disclosure of Interests: None declared

DOI: 10.1136/annrheumdis-2019-eular.731

\section{AB1262 THE RELATIONSHIP BETWEEN HYPERURICEMIA AND HEART RATE IRREGULARITY, USING DATA FROM THE KOREAN NATIONAL HEALTH AND NUTRITION EXAMINATION SURVEY 2016}

Yeonghee Eun ${ }^{1}$, Eun-Jung Park ${ }^{2}$, Ji-Young Chai ${ }^{3}$, Hoon-Suk Cha ${ }^{1}$, Eun-MI Koh ${ }^{1}$, Hyungjin Kim ${ }^{1}$, Jaejoon Lee ${ }^{1}$. 'Samsung Medical Center, Department of Medicine, Seoul, Korea, Rep. of (South Korea); ${ }^{2}$ National Medical Center, Department of Medicine, Seoul, Korea, Rep. of (South Korea); ${ }^{3}$ Bundang Jesaeng General Hospital, Department of Internal Medicine, Seongnam, Korea, Rep. of (South Korea)

Background: Hyperuricemia is one of the well-known cardiovascular risk factors. In recent studies, hyperuricemia has been shown to be an independent risk factor for atrial fibrillation, which may be associated with oxidative stress or inflammation. There is still a lack of data on the association of hyperuricemia and arrhythmia other than atrial fibrillation. Objectives: In this study, we investigated the relationship between hyperuricemia and heart rate $(\mathrm{HR})$ irregularity using representative sample data of adult Korean

Methods: The study included 5870 subjects aged 19 years or older who completed the uric acid measurement in the Korean National Health and Nutrition Examination Survey conducted in 2016. Logistic regression was used to analyze the association between hyperuricemia and HR irregularity identified by the examiner at the time of the survey.

Results: Subjects with HR irregularity were older, had more smoking and drinking, had a higher prevalence of HTN, and had lower glomerular filtration rate than those with regular heartbeat. In the presence of hyperuricemia, the HR irregularity was three times higher than in the absence (HR $3.65,95 \% \mathrm{Cl} 1.77-7.53, P=0.0005)$. The association of HR irregularity and hyperuricemia was significant in most subjects, especially in those older than 65 years, with diabetes and hypertension.

Conclusion: Hyperuricemia was highly correlated with HR irregularity in adult Korean representative sample data, especially in subjects with conventional cardiovascular risk factors such as old age, hypertension, diabetes mellitus. This study included all subjects who showed HR irregularity at the time of examination, not just atrial fibrillation. Therefore, it is suggested that hyperuricemia may be associated with other arrhythmia as well as atrial fibrillation. Further researches are warranted to clarify the relationship between hyperuricemia and arrhythmia and its mechanism.
Disclosure of Interests: None declared

DOI: 10.1136/annrheumdis-2019-eular.7783

\section{AB1263 C-REACTIVE PROTEIN LEVEL AS A MARKER FOR DYSLIPIDEMIA, DIABETES, AND METABOLIC SYNDROME: RESULTS FROM THE KOREAN NATIONAL HEALTH AND NUTRITION EXAMINATION SURVEY:}

Hyemin Jeong ${ }^{1}$, Yeoung Hee Eun ${ }^{2}$, In-Young Kim ${ }^{3}$, Eun-Jung Park ${ }^{4}$, Jaejoon Lee ${ }^{2}$ Hyungjin Kim ${ }^{2}$, Chan Hong Jeon ${ }^{1} .{ }^{1}$ Soonchunhyang University Hospital, Bucheon, Bucheon, Korea, Rep. of (South Korea); ${ }^{2}$ Samsung Medical Center, Seoul, Korea, Rep. of (South Korea); ${ }^{3}$ National Police Hospital, Seoul, Korea, Rep. of (South Korea); ${ }^{4}$ National Medical Center, Seoul, Korea, Rep. of (South Korea)

Background: High sensitivity C-reactive protein (hsCRP) C-reactive protein (CRP) is a marker of inflammation and increased hsCRP is reported in many diseases including cardiovascular disease, diabetes, metabolic syndrome, arthritis, and malignancies.

Objectives: This study evaluated the association between hsCRP and comorbidities.

Methods: A total of 5,887 (weighted $n=40,251,868$ ) participants age 19 or older from the 2016 Korean National Health and Nutrition Examination Survey were included for analysis. Weighted prevalence and odds ratio (OR) of comorbidities were analyzed according to the continuous variable of log hsCRP levels.

Results: Mean age was 46.7 and mean hsCRP levels were $1.23 \mathrm{mg} / \mathrm{L}$ Mean hsCRP levels were higher in participants with cardiovascular dis eases and cardiovascular risk factors, osteoarthritis, rheumatoid arthritis, pulmonary tuberculosis, and several cancers of gastric, colon, breast, and cervix than the general population. In multivariable analysis, hsCRP concentration was associated with increased prevalence of hypertriglyceride mia (OR 1.15, 95\% Cl 1.04-1.28, $\mathrm{p}=0.007$ ), diabetes (OR 1.20, 95\% $\mathrm{Cl} 1.05-1.37, \mathrm{p}=0.005)$, and metabolic syndrome (OR $1.22,95 \% \mathrm{Cl}$ 1.11-1.35, $p<0.001)$ after adjustment for socioeconomic and lifestyle characteristics. There was no significant association between hsCRP level and cancers.

Conclusion: hsCRP was associated with increased risk of dyslipidemia, diabetes, and metabolic syndrome in the general population.

Disclosure of Interests: None declared

DOI: 10.1136/annrheumdis-2019-eular.5244

\section{AB1264 THE RELATIONSHIP BETWEEN SERUM URIC ACID AND PULMONARY FUNCTION IN KOREAN ADULT POPULATION: DATA FROM THE KOREA NATIONAL HEALTH AND NUTRITION EXAMINATION SURVEY:}

Hyemin Jeong ${ }^{1}$, Yeoung Hee Eun ${ }^{2}$, In-Young Kim ${ }^{3}$, Eun-Jung Park ${ }^{4}$,

Hyungjin Kim², Jaejoon Lee ${ }^{2}$, Chan Hong Jeon'. ' Soonchunhyang University Hospital, Bucheon, Bucheon, Korea, Rep. of (South Korea); '2Samsung Medical Center, Seoul, Korea, Rep. of (South Korea); ${ }^{3}$ National Police Hospital, Seoul, Korea, Rep. of (South Korea); ${ }^{4}$ National Medical Center, Seoul, Korea, Rep. of (South Korea)

Background: Hyperuricemia is associated with several comorbidities. The association between uric acid and pulmonary function is still controversial. Objectives: The aim of this study was to evaluate the relationship between serum uric acid and pulmonary function in Korean adult population.

Methods: A total of 3,177 (weighted $\mathrm{n}=19,770,902$ ) participants aged 40 years or older from the 2016 Korean National Health and Nutrition Examination Survey were included and performed cross-sectional study. Results: Participant with hyperuricemia was older than participants with normouricemia in females. Body mass index, Mean arterial pressure, and hemoglobin $\mathrm{A} 1 \mathrm{c}$, and estimated glomerular filtration rate (eGFR) were significantly associated with uric acid levels in both sex. Hyperuricemia was significantly associated with decreased FEV1 and FVC in females after adjustment for age, income, region, education, marital status, alcohol consumption, smoking, body mass index, mean arterial pressure, hemoglobin $\mathrm{A} 1 \mathrm{c}$, and eGFR $(\beta=-0.143, \mathrm{P}$-value $=0.002$ and $\beta=-0.159, \mathrm{P}$-value $=$ 0.001 , respectively). There was no significant association between uric acid levels and lung function in males. After additional adjustment for respiratory disease including pulmonary tuberculosis, asthma, and lung cancer, hyperuricemia was associated with decreased FEV1 and FVC in females $(\beta=-0.142, P$-value $=0.001$ and $\beta=-0.161, P$-value $<0.001$, respectively)

Conclusion: Hyperuricemia was associated with decreased FVE1 and FVC in female general population. 